\title{
FRONTIERS OF NARRATIVE STUDIES
}

\section{EDITOR-IN-CHIEF}

Shang Biwu, Shanghai Jiao Tong University, China

\section{CO-EDITORS}

William Baker, Northern Illinois University, USA

Wolfgang G. Müller, Friedrich Schiller University, Jena, Germany

\section{EDITORIAL BOARD}

Péter Hajdu, Hungarian Academy of Sciences, Hungary

David Herman, Durham University, UK

Stefan Iversen, Aarhus University, Denmark

Karin Kukkonen, University of Oslo, Norway

Gunther Martens, Ghent University, Belgium

Brian McHale, Ohio State University, USA

Ansgar Nünning, University of Giessen, Germany

Vera Nünning, University of Heidelberg, Germany

Ruth E. Page, University of Birmingham, UK

James Phelan, Ohio State University, USA

Gerald Prince, University of Pennsylvania, USA

Qiao Guoqiang, Shanghai International Studies University, China Brian Richardson, University of Maryland, USA

Marie-Laure Ryan, Independent Scholar, USA

Dan Shen, Peking University, China

Michael Toolan, University of Birmingham, UK

Wang Ning, Shanghai Jiao Tong University, China

\section{DE GRUYTER}


ABSTRACTED/INDEXED IN Baidu Scholar · CNKI Scholar (China National Knowledge Infrastructure) - CNPIEC: cnpLINKer Dimensions EBSCO Discovery Service · Google Scholar · J-Gate · JournalTOCs · KESLI-NDSL (Korean National Discovery for Science Leaders) · Microsoft Academic - MLA International Bibliography · MyScienceWork · Naver Academic $\cdot$ Naviga (Softweco) · Primo Central (ExLibris) · Publons · QOAM (Quality Open Access Market) · ReadCube · Semantic Scholar · Sherpa/RoMEO · Summon (ProQuest) · TDNet · Ulrich's Periodicals Directory/ulrichsweb · WanFang Data $\cdot$ Web of Science: Emerging Sources Citation Index · WorldCat (OCLC) · Yewno Discover

ISSN 2509-4882 · e-ISSN 2509-4890

All information regarding notes for contributors, subscriptions, Open Access, back volumes and orders is available online at www.degruyter.com/journals/fns

RESPONSIBLE EDITOR Prof. Dr. Shang Biwu, English Department, Shanghai Jiao Tong University, 800 Dongchuan Road, Minhang District, Shanghai 200240, P.R. China, Tel.: +86-13661438182, E-Mail: biwushang@sjtu.edu.cn

\section{EDITORIAL ASSISTANTS}

Lin Yuzhen, Shanghai Jiao Tong University, China

Tang Yili, Shanghai Jiao Tong University, China

JOURNAL MANAGER Diana Kostovic, De Gruyter, Genthiner Straße 13, 10785 Berlin, Germany, Tel.: +49 (0)30 260 05-376, Fax: +49 (0)30 260 05-273, E-Mail: diana.kostovic@ degruyter.com

RESPONSIBLE FOR ADVERTISEMENTS Markus Kügel, De Gruyter, Rosenheimer Str. 143, 81671 München, Germany. Tel.: +49 8976 902-424, e-mail: anzeigen@ degruyter.com.

(C) 2021 Walter de Gruyter GmbH, Berlin/Boston

PRINTING Franz X. Stückle Druck und Verlag e.K., Ettenheim

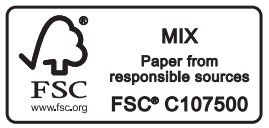




\section{Contents}

Jiayi Chen and Marie-Laure Ryan

Storyworld, transmedia storytelling, and contemporary narrative theory:

An interview with Marie-Laure Ryan — 147

Seán Hand

Being for every other: Levinas in the anthropocene — 156

Dana Ryan Lande

Edmund Wilson, the Dead Sea Scrolls, and the rhetoric of protection - 176

Xiaomeng Wan

Corporeal narrative of insatiable desire in David Foster Wallace's

Infinite Jest — 193

Manali Karmakar and Bhuvaneswari G.

“The old boys' network": Medical space, gendered memory, and prejudices

in Sidney Sheldon's Nothing Last Forever — 207

Nathan D. Frank

I, theorist: Accrediting the "wild imagination" of Northanger Abbey -222

\section{Review}

Xinyi Cao

Bob Fischer, ed. The routledge handbook of animal ethics - 239 
